\title{
Instabilities and diffusion in a hydrodynamic model of a fluid membrane coupled to a thin active fluid layer
}

\author{
Niladri Sarkar and Abhik Basu \\ Theoretical Condensed Matter Physics Division, Saha Institute of \\ Nuclear Physics, 1/AF, Bidhannagar, Calcutta 700 064, India
}

(Dated: March 27, 2018)

\begin{abstract}
We construct a coarse-grained effective two-dimensional $(2 d)$ hydrodynamic theory as a theoretical model for a coupled system of a fluid membrane and a thin layer of a polar active fluid in its ordered state that is anchored to the membrane. We show that such a system is prone to generic instabilities through the interplay of nonequilibrium drive, polar order and membrane fluctuation. We use our model equations to calculate diffusion coefficients of an inclusion in the membrane and show that their values depend strongly on the system size, in contrast to their equilibrium values. Our work extends the work of S. Sankararaman and S. Ramaswamy [Phys. Rev. Lett., 102, 118107 (2009)] to a coupled system of a fluid membrane and an ordered active fluid layer. Our model is broadly inspired by and should be useful as a starting point for theoretical descriptions of the coupled dynamics of a cell membrane and a cortical actin layer anchored to it.
\end{abstract}

\section{INTRODUCTION}

Theoretical studies of equilibrium properties (both static and time-dependent) of fluid membranes are extensive and well-established by now [1-3]. Mechanical properties of membranes are essentially controlled by a few parameters, such as the membrane tension, bending modulus and spontaneous curvature [1]. These parameters completely characterise membrane fluctuation spectra at thermal equilibrium. By contrast, theoretical studies of the dynamical properties of driven fluid membranes are relatively at early stages of development. In this article, we consider the coupled dynamics of a single-component fluid membrane and an active fluid layer anchored to it, within a coarse-grained hydrodynamic approach. Apart from its direct theoretical interests, there are biological implications as well. Studies of biomembranes are important in cell biology context, since all cells are covered by cell membranes which typically have complicated internal structures [4]. At a coarse-grained level, however, cell membranes are usually described as a bilayer fluid (with complicated microscopic internal structures, see, e.g., [4, 5]). In eukaryotic cells, there is a thin layer of cross-linked actin filaments anchored to the membrane and associated with the cell cytoskeleton in typical eukaryotic cells. Knowledge of the properties of fluid membranes in equilibrium are not sufficient to characterise real biological membranes, such as plasma membranes of any cell, which are inherently nonequilibrium active systems [4, 6, 7]. For instance, cell membranes are constantly maintained out of equilibrium due to nonequilibrium processes of various types. It has now been realized that such nonequilibrium behaviour may underlie aspects of biomembrane dynamics [8, 9] , previously attributed purely to equilibrium thermal fluctuations. These nonequilibrium aspects affect experimental measurements of physical quantities characterising cell membrane dynamics, e.g., one finds anomalous diffusion [7, 10] (however, these are primarily due to coralling and inhibiting effects, different from active fluid effects discussed here). It is now generally accepted that such complex dynamical behaviour have their origins in generic nonequilibrium drives. In a living cell, there may be several different sources for such nonequilibrium behaviour - it could be due to active proteins inside the membrane that are often Adenosine Triphosphate (ATP) consuming enzymes, or the membrane could be driven out of equilibrium by the nonequilibrium fluctuations of an active cortical actin layer anchored to the membrane. The former case has been studied both experimentally and theoretically in details, see, e.g., Ref. [11]. The latter case is discussed in Refs. [12, 13]. In particular, Ref. [12] introduced a model that couples a membrane with actin polymerisation and contractile forces due to molecular motors, e.g., myosin. For a configuration of actin filaments which are typically normally directed to the membrane, Ref. [12] has shown how motor activities lead to generic transverse membrane waves. Ref. [13] discusses the influence of actin filament elasticity and retrograde flow on the force-velocity relations of motile cells. A related problem is the diffusion of small inclusions embedded in membranes, which is challenging both theoretically and experimentally. This has been extensively studied. For instance, Ref. 14] theoretically shows how curvature fluctuations (both thermal and non-thermal) in a membrane leads to enhancement of the effective thickness, that in turn causes a reduction in the measured diffusion coefficient. In a related work, Ref. [15] discusses how membrane fluctuations, both static or quenched and annealed or dynamic, affect the diffusion coefficient. By using a mixture of analytical and numerical methods they find that for a membrane with quenched fluctuations, the diffusion coefficient is substantially reduced by the quenched roughness, and is nicely described by an area-scaling law proposed there. In contrast, for the annealed case with small bending rigidity, the reduction in the the diffusion coefficient is less than in the quenched case. These studies on diffusion coefficients, however, do not include any nonequilibrium effects that arise due to the active stress (see below) in the active fluid 
layer anchored to the membrane. Elucidation of the generic effects of the active stress present in the ordered active fluid layer on the statistical properties of the attached fluid membrane is a major purpose of the present study.

Although a cell cortex is typically not in an ordered state for the polarisation degrees of freedom (the actin filaments), we consider an ordered state and study fluctuations about it. In particular, we assume the ordering direction to be parallel to the membrane. The other possible ordered state, viz where the actin filaments are predominantly perpendicular to the membrane is important as well (see, e.g., Ref. [12] for a study with perpendicular configuration of the filaments). Since a prime motivation of this work is to theoretically study in a general set up the effects of the nonequilibrium dynamics and fluctuations of an ordered layer of polar active fluid on a fluid membrane that is in contact with the active fluid layer, we have chosen to work with the parallel orientation as an illustrative example. Nevertheless, our scheme of calculations may be easily extended to filaments with perpendicular ordering. We briefly discuss this later. Our work here will have broad relevance and act as a starting point for more detailed study of the dynamics of a single-component fluid membrane in contact with a thin layer of cortical actin filaments. Recently, artificial actin layers attached to a fluid membrane have been constructed and experiments performed on them [16, 17]. Experiments include investigation of the structure of the actin layer and measurement of diffusion of inclusions in the membrane. The latter experiments do find rising lateral diffusion coefficients for larger systems [16]. While preparation of and experimental studies on artificial actin layers of a particular macroscopic configuration (i.e., a particular ordered state) anchored to a membrane is definitely technically a difficult task, some of our results described here may in principle be tested by performing controlled experiments on such systems.

In this article, a thin cortical layer of actin filaments is considered at length scales much larger than the filament lengths and layer thickness with polar ordering, for which a generic coarse-grained continuum two-dimensional $(2 d)$ description would be appropriate. Further, we consider time scales larger than the unbinding time scale of the cross-linking proteins of the actin filaments, so that the actin network behaves like a fluid. In order to model the nonequilibrium dynamics of cortical actin layer, we use the active fluid description, proposed and elucidated in Refs. [18, 19]. Such approaches have been successfully used in a variety of driven systems, see, e.g., Refs. 20 23. for extensive discussions about the subject. There have been a number of studies on the properties of active fluid in confining thin geometries before. For example, Refs. [24, 25] proposed a mechanism for development of contractile rings in cell cortex. Ref. 25] discussed large scale flows of the acto-myosin cortex in Caenorhabditis elegans zygotes. Further, Ref. [26] provides a one-dimensional (1d) model for pattern formation in active fluids (see also Ref. [27] for a $1 d$ model of patterns in a cell cortex). In addition, Ref. [28] points out the existence of universal dynamic patterns by lateral membrane waves in motile cells. Our results here are complementary to those mentioned above. In this article, we systematically derive a set of effective $2 d$ coarse-grained hydrodynamic equations of motion for the membrane height field, local orientational order parameter, concentration and the velocity fields, which we use to obtain results characterising fluctuations and correlations in the system. In particular, we show that there are generic moving or static instabilities (i.e., with or without propagating modes). We use a stochastically driven versions of these to illustrate the effects of the active (nonequilibrium) dynamics on the lateral and rotational diffusion coefficients of an inclusion in the membrane. We find that under certain situations there could be non-trivial system size dependences of the diffusion coefficients in comparison with their equilibrium expressions. The rest of this article is organised as follows: In Sec. II] we describe the basic model considered here and set up the equations of motion. We then show in Secs. ПIA and ПIB that these equations, upon linearisation show emergence of linear instabilities when the strength of the nonequlibrium drive, denoted by $\Delta \mu$ exceeds a critical value. Finally, in Sec. IV we calculate lateral and rotational diffusion coefficients of an inclusion in the membrane; we show that for a membrane-active fluid combine lateral and rotational diffusion coefficients depend on the system size in ways that are very different from the known results for diffusion in systems at equilibrium. We summarise and discuss our results in the context of existing experimental results in Sec. $\nabla$ ]

\section{MODEL SYSTEM AND EQUATIONS OF MOTION}

For the sake of simplicity, we analyse here the effect of activity on the fluctuation spectrum of a planar (flat on an average) membrane only. Our calculational framework as we formulate below is general. However, we use it to study, within a linearised approach, typically the properties of perturbations about an ordered, uniform reference state of the active fluid.The construction of our model is generally inspired by the structure of a real eukaryotic cell. There is a thin layer of cortical actin or cell cortex anchored to the cell membrane in an eukaryotic cell. This layer is rich in actin filaments which may be locally preferentially orientated either parallel or perpendicular to the membrane. The bulk of the cell is the cytoplasm consisting of cytoskeleton and other cellular organelles. We model the cortical actin layer by a thin film of active fluid of viscosity $\eta$, thin in the $z$-direction and spread along the $x y$ plane with active polar particles (since the actin filaments are polar) suspended in it. In our discussions below we only consider a macroscopically ordered state of the active particles (actin filaments) with in-plane ordering. We do 
not discuss the other possible case of perpendicular orientations in details here. The active fluid is assumed to be anchored to one side of a fluid membrane characterised by a bending stiffness. We consider two distinct versions of our system: (i) Model I: The fluid membrane-active fluid combine is embedded inside a bulk isotropic fluid on both sides. The bulk fluid viscosity $\eta^{\prime}$ is assumed to be much smaller than the active fluid viscosity (for simplicity; see below), and (ii) Model II: The system rests on a solid substrate below. The two cases are physically different: First of all, the presence of the solid substrate introduces friction and, as a result, the momentum (same as the hydrodynamic velocity $\mathbf{v}$ for an incompressible system) is no longer a conserved field. In contrast, when there is a bulk fluid surrounding the system there is no friction at the interface and hence $\mathbf{v}$ remains a conserved variable. This leads to long-ranged hydrodynamic interactions in such a system. Secondly, the presence of the solid substrate breaks the full three-dimensional (3d) rotational invariance of the problem, where as, when there is an embedding bulk fluid, the system is invariant under the full $3 d$ rotational invariance (see below). This has important ramifications on the possible structure of the membrane free energy, as we will discuss below. Despite the cell biological inspiration for our work, the connections between our model and the structure of an eukaryotic cell is not very strict, and thus our model and results cannot be directly applied to a biologically relevant system: First of all, the cortical actin layer does not exist in an ordered state with in-plane orientation; at the scale of the cell it is generally isotropic. Secondly, in a real cell, there is not any sharp dividing surface separating the cortex from the bulk. Although our idealised model is not accurate for a real cell, nevertheless it allow us to obtain interesting and non-trivial result in a simple set up, highlighting how the environment (in the form of a solid substrate below or a passive, isotropic embedding fluid around the system) may drastically affect macroscopic physical properties. Our work may be considered as a starting point of more realistic calculations. Our treatment generalises Ref. [29] to the case of a coupled system of a fluid membrane and an active fluid film. (See also Ref. [30] for discussions on the effects of a solid substrate on the dynamics of thin liquid film covered by a membrane.) We now discuss the constructions of Model I and Model II in some details below.

\section{A. Model I: System covered by isotropic fluid on both sides}

Consider first our Model I, where the fluid membrane-active fluid layer combine is embedded by an isotropic fluid on both sides. In order to keep the ensuing algebra tractable and to obtain closed equations and non-trivial results without having to introduce too many details we make a number of simplifying assumptions which we now discuss. First of all, we assume $\eta \gg \eta^{\prime}$. To set up the context for our work, we refer to the well-known results in Ref. [31], where the translational diffusivity of an inclusion is calculated in a thin $2 d$ inflexible flat layer of an isotropic viscous fluid of viscosity $\eta$ confined between bulk isotropic viscous fluids of viscosity $\eta_{1}\left(\eta_{1} / \eta\right.$ finite) on both sides. We here consider a thin active fluid layer containing orientational degrees of freedom in their ordered state and covered by a fluid membrane on one side of finite stiffness. However, we consider only the special limit of $\eta \gg \eta^{\prime}$. Thus our work here can be thought of as generalisation of and complementary to Ref. 31.

In order to specify the problem completely, we impose the following boundary conditions: When the fluid membraneactive fluid layer is covered by an isotropic fluid on both sides, the boundary conditions are as follows: (i) At the interfaces $\left(z=h_{1}\right.$ and $\left.h_{2}\right)$ we impose $\mathbf{p}$ to be parallel to the local tangent plane on the surfaces, i.e., $\mathbf{p} \cdot \hat{N}=0$ at $z=h_{1}$ and $h_{2}$, as the current of active particles is along $\mathbf{p}$ and the particles cannot leave the film, where $\hat{N}$ is the local normal at $h_{1}$ and $h_{2}$, and (ii) continuity of the shear stress at $z=h_{1}$ and $h_{2}$. The free energy functional $\mathcal{F}_{p}$ of the system is a functional of $h_{1}, h_{2}$ and $\mathbf{p}$. The form of $\mathcal{F}_{p}$ may be inferred from symmetry considerations. It must generally be invariant under an arbitrary tilt (equivalently a rotation) $h_{1,2} \rightarrow h_{1,2}+\mathbf{a} \cdot \mathbf{x}$ of the free surfaces, where $\mathbf{a}$ is an arbitrary $3 d$ vector and $\mathbf{x}$ is a $3 d$ radius vector. This ensures that the most leading order (in gradients) coupling bilinear in $\mathbf{p}$ and $h_{1}$ or $h_{2}$ could be of the form $\boldsymbol{\nabla} \cdot \mathbf{p} \nabla^{2} h_{1,2}$. This is a polar term, since it has no $\mathbf{p} \rightarrow-\mathbf{p}$ symmetry. Further, it violates $h_{1,2} \rightarrow-h_{1,2}$ symmetry as well, which is admissible since the actin filaments are anchored only on one side of the membrane. For the polar order parameter, we use the Frank free energy [32] in the limit of equal Frank's constants, denoted by $D$ here. Assuming that the surface tension of the membrane is negligible, the general form of the free energy functional of the combined system of membrane (top), free surface (bottom) and active fluid is given by (in the Monge gauge [35])

$$
\begin{aligned}
\mathcal{F}_{L}\left[h_{1}, h_{2}, \mathbf{p}\right] & =\frac{1}{2} \int d^{2} r\left[\sigma\left(\nabla_{\perp} h_{2}\right)^{2}+\kappa\left(\nabla_{\perp}^{2} h_{1}\right)^{2}\right] \\
& +\frac{1}{2} \int d^{2} r \int_{h_{2}}^{h_{1}} d z\left[\hat{C}(\boldsymbol{\nabla} \cdot \mathbf{p})\left(\nabla^{2} h_{1}\right) \delta\left(z-h_{1}\right)+\hat{C}(\boldsymbol{\nabla} \cdot \mathbf{p}) \nabla^{2} h_{2} \delta\left(z-h_{2}\right)+D\left(\nabla_{i} p_{j}\right)^{2}\right],
\end{aligned}
$$

where $\kappa$ is the bending rigidity of the membrane, $\sigma$ the interfacial tension of the surface at $h_{2}, \hat{C}$ is a coupling constant that couples the orientational field $\mathbf{p}$ with the fluctuations of the height fields. In principle, one needs to solve for 
the dynamics of $h_{1}$ and $h_{2}$ separately for a full dynamical description of the problem. Operators $\nabla_{\perp}=\left(\frac{\partial}{\partial x}, \frac{\partial}{\partial y}\right)$ and $\nabla=\left(\frac{\partial}{\partial x}, \frac{\partial}{\partial y}, \frac{\partial}{\partial z}\right)$ are the $2 d$ and $3 d$ Laplacians, respectively. At this stage, in order to simplify the ensuing algebraic manipulations, but still be able to display the dramatic effects of the hydrodynamic interactions, we consider a special simplifying (but admittedly artificial) limit of the problem with $\sigma$ being large enough (formally a diverging $\sigma$ ), such that fluctuations of $h_{2}$ are suppressed and may henceforth be ignored. Thus we are required to solve for the dynamics of $h_{1}$ only (along with $\mathbf{p}$ and $c$ ). It must be mentioned that the limit of large $\sigma$ is primarily of theoretical interests and is not expected to be observed in any real (biological) system. Despite these limitations of our assumption of large $\sigma$, we obtain interesting results which are expected to be present qualitatively even with finite $\sigma$. In order to set notations simpler, we set $h_{1}=h$ and $h_{2}=0$ in our subsequent analysis below. Thus $h_{2}$ drops out of the dynamics; see Fig. (11) for a schematic picture of our Model I system. Consequently, the free energy (1) reduces to

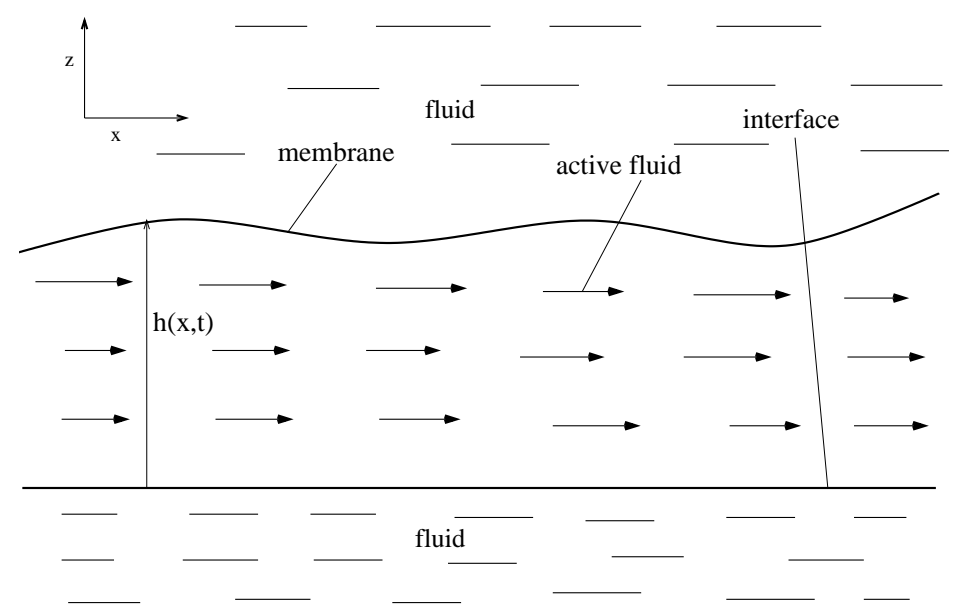

FIG. 1: A schematic diagram of our model system showing the membrane and the active fluid layer. The arrows indicate the direction of macroscopic orientation (here along the $x$-axis).

$$
\begin{aligned}
\mathcal{F}_{L}[h, \mathbf{p}] & =\frac{1}{2} \int d^{2} r\left[\kappa\left(\nabla_{\perp}^{2} h\right)^{2}\right] \\
& +\frac{1}{2} \int d^{2} r \int_{0}^{h} d z\left[\hat{C}(\nabla \cdot \mathbf{p})\left(\nabla^{2} h\right) \delta(z-h)+D\left(\nabla_{i} p_{j}\right)^{2}\right]
\end{aligned}
$$

It is invariant under $h \rightarrow h+\mathbf{a} \cdot \mathbf{x}$. Now imposing the kinematic boundary conditions [33] on $v_{z}$ at $h$ for an impermeable membrane we write:

$$
\frac{\partial h}{\partial t}+\mathbf{v}_{\perp} \cdot \nabla_{\perp} h=v_{z},
$$

which connects the height fields $h$ with the hydrodynamic velocity field $\mathbf{v}=\left(v_{x}, v_{y}, v_{z}\right)$ at the location of the membrane $(z=h)$. Here, $\mathbf{v}_{\perp}=\left(v_{x}, v_{y}\right)$. In the expression (2) above, when $D-\hat{C}^{2} / \kappa>0$, the equilibrium phases are spatially uniform, else modulated phases are possible. In this article we consider $D-\hat{C}^{2} / \kappa>0$, corresponding to uniform equilibrium phase only [34]. Thus finally the relevant slow modes in this effective $2 d$ problem are (i) a concentration of active polar particles $c(\mathbf{r}, t)$, (ii) a local orientation field (describing the local orientation in the cortical actin) given by $\mathbf{p}(\mathbf{r}, t)$, and (iii) a local height fields $h(\mathbf{r}, t)$ to describe the nearly flat membranes (in the Monge gauge [35]), where $\mathbf{r}=(x, y)$ is the in-plane coordinate and $t$ is the time. We take the $x y$-plane as the easy plane for polarisation fluctuation. Further, we consider macroscopic orientational order given by a reference state $\mathbf{p}=(1,0,0)$ i.e. we have macroscopic ordering along the $x$ axis and impose fixed length constraint on $\mathbf{p}: p^{2}=1$. For an active system, the polarity implies a current $v_{0} c \mathbf{p}$ with respect to the fluid, where $v_{0}$ is a characteristic drift velocity.

The relevant intrinsic stress field of the active particles is given by [20, 22, 29]

$$
\sigma_{i j}^{a}=\Delta \mu c(\mathbf{r}) p_{i}(\mathbf{r}) p_{j}(\mathbf{r})
$$

which is of nonequilibrium origin. Stress $\sigma_{i j}^{a}$ is said to be contractile or extensile for the constant $\Delta \mu<0$ or $\Delta \mu>0$. The generalised Stokes Equation for $\mathbf{v}$, is obtained by using the force balance condition after neglecting inertia as 
appropriate for small masses in typical biological systems. Velocity components $\mathbf{v}_{\perp}$ are to be solved from the full Stokes equation

$$
\eta \nabla_{\perp}^{2} \mathbf{v}_{\perp}+\eta \partial_{z}^{2} \mathbf{v}_{\perp}-\nabla_{\perp} \Pi-\nabla_{j} \sigma_{\perp j}^{a}=0
$$

where $\sigma_{\perp j}^{a}=\left(\sigma_{x j}^{a}, \sigma_{y j}^{a}\right)$. At the membrane $(z=h)$ we have $\mathbf{p} \cdot \hat{N}=0$ where $\hat{N}$ is the local normal at $h(x, y)$. By using the Monge gauge for the membrane we have $\hat{N}=\left(-\nabla_{\perp} h, 1\right) / \sqrt{1+\left(\nabla_{\perp} h\right)^{2}}$ to be the outward normal to the membrane surface. This gives us

$$
p_{z} \simeq \frac{\partial_{x}(h)}{h} z .
$$

This then yields $\partial_{z} p_{z} \simeq \frac{1}{h} \partial_{x} h$. In order to solve for $\mathbf{v}$ from the above equations we must first find out $\nabla \cdot \sigma^{a}$. Within our linearised treatment, assume $\mathbf{p}_{\perp}=\hat{x}+\theta \hat{y}$ with small $\theta$, where $\mathbf{p}_{\perp}=\left(p_{x}, p_{y}\right) \sim(1, \theta)$ to the lowest order. With $|\theta| \ll 1$ considering small fluctuation, we may obtain different components of $\nabla_{j} \sigma_{i j}^{a}$. To eliminate $\Pi$ we solve the Stokes equation for $v_{z}$ in the lubrication approximation, i.e., we write $\partial_{z} \Pi=-\partial_{i} \sigma_{i z}^{a}=-\Delta \mu \frac{c_{0}}{2} \partial_{x}^{2} h$. This yields

$$
\Pi(x, y, z, t)=P_{0}+\Delta \mu \frac{c_{0}}{2}(h-z) \partial_{x}^{2} h-f(h),
$$

where $f(h)=-\frac{\delta \mathcal{F}_{\mathcal{L}}}{\delta h}=-\kappa \nabla_{\perp}^{4} h+\hat{C} \nabla_{\perp}^{2} \nabla_{\perp} \cdot \mathbf{p}_{\perp}$ is the elastic force of the membrane. Equation (7) tells us that the balance of the normal component of stress at the membrane gives the condition of balancing the fluid stress by the elastic force of the membrane (2). Thus the active contribution to the pressure in (7) comes with the same signature of $\Delta \mu$ as in the active stress expression (4).

The solutions of equation (5) are facilitated greatly in terms of the in-plane Fourier transformation: For a system as above, the general form for the hydrodynamic kernel is $1 /\left(\eta q^{2}+\eta^{\prime} q / h_{0}\right)$ where $h_{0}$ is the average thickness of the system, where $\eta$ and $\eta^{\prime}$ are the viscosities of the active fluid and the surrounding passive isotropic fluid, respectively. Thus for $q>\eta^{\prime} /\left(\eta h_{0}\right)$, the hydrodynamic kernel is identical to that of a free standing system. If (for the sake of simplicity) we now assume that $\eta \gg \eta^{\prime}$, then in a broad window of wavevector, the hydrodynamic kernel may be approximated by $1 / \eta q^{2}$. Further in that limit, the continuity of the shear stress at $z=h$ and $z=0$ actually implies vanishing of the shear stress at $z=h$ and $z=0$. Hence, in order to solve for $v_{x}$ and $v_{y}$, we now need to solve Eq. (5) subject to boundary conditions of zero shear stress at $z=h$ and $z=0$. To the leading order in smallness this translates into

$$
\eta \frac{\partial v_{x}}{\partial z}+\Delta \mu p_{z}=0, \quad \eta \frac{\partial v_{y}}{\partial z}=0
$$

at $z=h$ and

$$
\eta \frac{\partial \mathbf{v}_{\perp}}{\partial z}=0
$$

at $z=0$ ( $\operatorname{since} p_{z}=0$ strictly at $z=0$ ). Thus, for this wavevector range, effectively we have a free standing active fluid, covered on one side by a fluid membrane and a free surface on the other. In what follows below we stay in this limit only [36]. Thus

$$
\begin{gathered}
v_{y}=-i \frac{q_{y}}{\eta q^{2}}\left[-\frac{3 c_{0} \Delta \mu h_{0}}{4} q_{x}^{2} h+\kappa q^{4} h-i \hat{C} q_{y} q^{2} \theta\right]-i \frac{c_{0} \Delta \mu}{\eta q^{2}} q_{x} \theta+f_{y}^{L}, \\
v_{x}=-\frac{1}{\eta q^{2}}\left[-i \frac{3 \Delta \mu h_{0} c_{0}}{4} q_{x}^{3} h+i \kappa q_{x} q^{4} h+\hat{C} h_{0} q^{2} q_{x} q_{y} \theta+i\left(\Delta \mu q_{x} c+c_{0} \Delta \mu q_{y} \theta+\frac{2 c_{0} \Delta \mu}{h_{0}} q_{x} h\right)\right]+f_{x}^{L},
\end{gathered}
$$

for $q h_{0} \ll 1$ (we work in this limit which enables us to continue using the Lubrication approximation). Here we have performed an in-plane Fourier transform with $\mathbf{q}$ as the Fourier conjugate of $\mathbf{r}$. Functions $f_{i}^{L}, i=x, y$ are zero-mean, Gaussian noises with variances $\left\langle f_{i}^{L}(\mathbf{q}, \omega) f_{j}^{L}(-\mathbf{q},-\omega)\right\rangle=\frac{2 K_{B} T}{\eta q^{2}} \delta_{i j}$. We have considered thermal noises for simplicity, although in real biological situations there are non-equilibrium noises as well.

From the incompressibility of the fluid we obtain $v_{z}=-\int \nabla_{\perp} \cdot \mathbf{v}_{\perp} d z$ together with the condition $v_{z}=0$ at $z=0$. Neglecting terms with higher derivatives and linearising about $\langle h\rangle=h_{0}$ and $\langle c\rangle=c_{0}$ and denoting $h$ and $c$ as the height and concentration fluctuations from their respective averages we obtain by using Eqs. (10) and (11)

$$
\begin{aligned}
\frac{\partial h}{\partial t}=v_{z}(z=h) & =\frac{1}{\eta q^{2}}\left[\frac{3 \Delta \mu c_{0} h_{0}^{2}}{4} q^{2} q_{x}^{2} h+i \hat{C} h_{0} q^{4} q_{y} \theta-\kappa h_{0} q^{6} h-\left(\Delta \mu h_{0} q_{x}^{2} c\right.\right. \\
& \left.\left.+2 c_{0} h_{0} \Delta \mu q_{x} q_{y} \theta+2 \Delta \mu c_{0} q_{x}^{2} h\right)\right]+\xi_{h}
\end{aligned}
$$


in the Fourier space for a free standing system, $\xi_{h}$ is a zero-mean Gaussian white noise related to $f_{i}^{L}$ and hence with a variance $\frac{2 K_{B} T h_{0}^{2}}{\eta}$.

Dynamics of the polar orientation field $\mathbf{p}$ differ from that of the more usual nematic director in that the equation of motion of $\mathbf{p}$ now must include terms [37] which violates the $\mathbf{p} \rightarrow-\mathbf{p}$ symmetry of the nematic director field. Apart from the usual terms [37] we can have a symmetry-permitted spontaneous splay term [29] in the free energy functional like $\mathcal{F}_{s p} \equiv-\int d^{3} x \tilde{C} \boldsymbol{\nabla} \cdot \mathbf{p}$, where $\tilde{C}$ is a parameter which depends on the concentration of activity i.e. $\tilde{C}=\tilde{C}\left(c_{0}\right)+\tilde{C}^{\prime}\left(c_{0}\right) \delta c+\ldots \ldots \equiv C+C^{\prime} \delta c$. Such terms may arise essentially due to the generic structural differences between the head and tail of the polar molecules. These will contribute $-\Gamma \frac{\delta \mathcal{F}_{L}}{\delta \mathbf{p}}=-\Gamma C^{\prime} \nabla c$ to the equation of motion for $\mathbf{p}, \Gamma$ being a kinetic coefficient. The $3 d$ equation of motion for $p_{y}=\theta$ becomes

$$
\partial_{t} \theta=-a_{1} v_{0} \partial_{x} \theta-\xi \partial_{y} c+\hat{C} \nabla^{2} \partial_{y} h \delta(z-h)+\left(\lambda A_{y x}-\Omega_{y x}\right)+D \nabla^{2} \theta+\xi_{\theta},
$$

where $\xi=\Gamma C^{\prime}, A_{i j}=\frac{1}{2}\left(\nabla_{i} v_{j}+\nabla_{j} v_{i}\right)$ is the strain rate tensor and $\Omega_{i j}=\frac{1}{2}\left(\nabla_{i} v_{j}-\nabla_{j} v_{i}\right)$ is the vorticity tensor. The first term of Eq. (13) represents the advection of polar particles with the embedding fluid. The coefficient $a_{1}$ is not necessarily unity, due to the lack of Galilean invariance. The next two terms are coupling terms coupling $\theta$ with the gradient of concentration of the active particles and the fluctuation of the membrane surface respectively. Coupling constant $\lambda$ couples flow (strain rate tensor) with the local orientation. For stable flow-alignment $|\lambda|>1$ [32]. The last term is the diffusion term representing the Frank free energy [26] contribution to the dynamics in the limit of equal Frank's constants. Noise $\xi_{\theta}$ is zero-mean, Gaussian distributed with a variance $\frac{2 K_{B} T}{\eta}$ [38]. After eliminating $v_{x}$ and $v_{y}$ by using Eqs (11) and (10), under $z$-averaging yields an equation for $\theta$, given by equation (35), as given in the Appendix (see Sec. VI) which depends only on $h, \theta$ and $c$. When there is a solid substrate below, $\theta$ follows the same equation as (13), except the tilt-polarisation coupling term now reads $\hat{C} \partial_{y} h$.

In our system the drift velocity of the polar particles with respect to the fluid is $v_{0}$ and hence the current due to active particles is given by $v_{0} c \mathbf{p}$. Thus the continuity equation apart from diffusion is given by $\partial_{t} c+\nabla \cdot\left[\left(\mathbf{v}+v_{0} \mathbf{p}\right) c\right]=0$. Now linearising about $c=c_{0}+c$ and using incompressibility we get

$$
\partial_{t} c=-i v_{0} c_{0} q_{y} \theta-i v_{0} q_{x} c+O\left(q_{x}^{2}, q_{y}^{2}\right) .
$$

\section{B. Model II: Membrane-active fluid combine rests on a solid substrate}

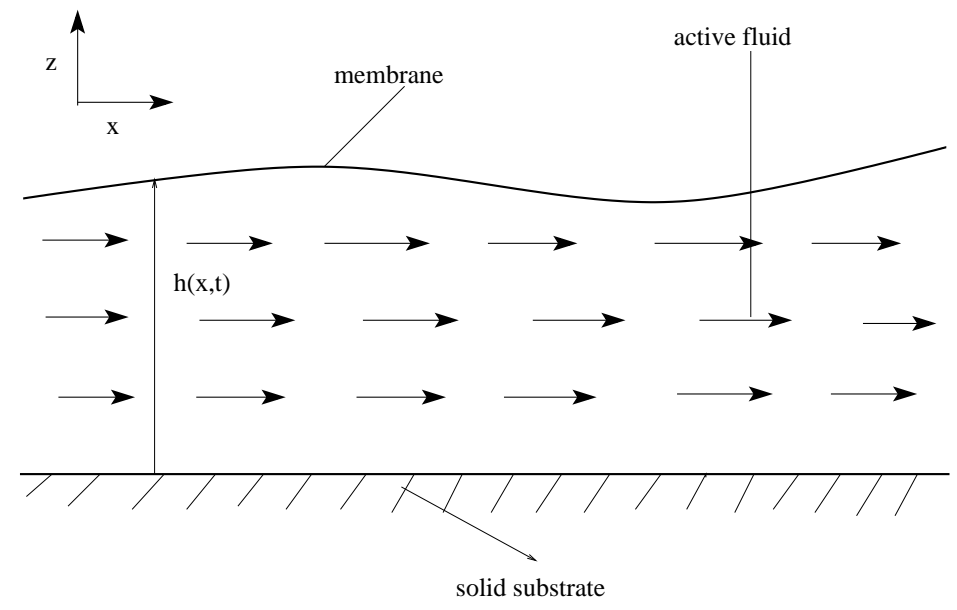

FIG. 2: A schematic diagram of our model system showing the membrane and the active fluid layer. The arrows indicate the direction of macroscopic orientation (here along the $x$-axis).

We now briefly discuss setting up the equations of motion when the fluid membrane-active fluid layer combine rests on a fully flat solid substrate; see Fig. (2) for a pictorial representation. Model II is important in the context in cell locomotion on a rigid substrate and lamellipodium. A lamellipodium is a cytoskeletal projection on the mobile edge of a cell. It is effectively a quasi- $2 d$ network of actin filaments and is responsible for cell movement along a solid substrate (see, e.g., Ref. [4] for a general description of this). We essentially follow the line of arguments and calculations of the previous section, differing only in some details. First of all, in the present case we are concerned with the dynamics of the top fluid membrane (at $z=h$ ) - active fluid layer combined, where as the bottom solid surface (at $z=0$ ) is 
fixed. The latter is the preferred frame of reference for this model (hereafter Model II). This breaks the $3 d$ rotational invariance discussed for Model I above. This consideration then dictates the form of the free energy $\mathcal{F}_{S}$ as

$$
\mathcal{F}_{S}[h]=\frac{1}{2} \int d^{2} r \kappa\left(\nabla_{\perp}^{2} h\right)^{2}+\frac{1}{2} \int d^{2} r \int_{0}^{h} d z\left[\hat{C} \mathbf{p} \cdot \nabla h \delta(z-h)+D\left(\nabla_{i} p_{j}\right)^{2}\right],
$$

where, as before in (2),$\kappa$ is the bending rigidity of the fluid membrane. Note that the bilinear coupling term in $h$ and $\mathbf{p}$ is more relevant (in a renormalisation group sense) than the corresponding terms in (2). We continue to denote the coupling constant by $\hat{C}$. Further we use the equal Frank's constant limit, represented by $D$, for the Frank free energy. The pressure $\Pi$ may be simply evaluated as above; we obtain

$$
\Pi=\Delta \mu \frac{c_{0}}{2}(h-z) \partial_{x}^{2} h+\kappa \nabla_{\perp}^{4} h-\hat{C} \nabla_{\perp} \cdot \mathbf{p}_{\perp}
$$

where we have used the boundary condition $\Pi=0$ at $z=0$ (setting the zero of pressure) and balanced the normal component of stress at $z=h$. Velocity components $v_{x}$ and $v_{y}$ may be calculated from the Stokes Equation. For no-slip boundary conditions for $\mathbf{v}_{\perp}$ at $z=0$, which we impose here, Stokes equation for $\mathbf{v}_{\perp}$ may be written in the lubrication approximation $\left(\left|\nabla_{\perp} \mathbf{v}_{\perp}\right|<<\left|\partial_{z} \mathbf{v}_{\perp}\right|\right)$

$$
\eta \partial_{z}^{2} \mathbf{v}_{\perp}-\nabla_{\perp} \Pi-\nabla_{j} \sigma_{\perp j}^{a}=0
$$

Now use (16) for $\Pi$ in Eq. (17) to calculate $v_{x}$ and $v_{y}$ : Equation (17) may be twice integrated with respect to $z$ to obtain $v_{x}$ and $v_{y}$. The ensuing constants of integration are to be eliminated by using the boundary conditions $v_{x}=0=v_{y}$ at $z=0$ and $\partial_{z} v_{y}=0, \partial_{z} v_{x}+\Delta \mu p_{z}\left(\mathbf{r}_{\perp}, z=h\right)=0$ at $z=h$. The resulting equations of motion for $\mathbf{v}_{\perp}=\left(v_{x}, v_{y}\right) \mathrm{read}$

$$
\begin{aligned}
\mathbf{v}_{\perp}= & \Delta \mu \frac{c_{0}}{2 \eta}\left(\frac{z^{2}}{2}-h z\right) \nabla_{\perp}\left(h \partial_{x}^{2} h\right)-\Delta \mu \frac{c_{0}}{4 \eta}\left(\frac{z^{3}}{3}-h^{2} z\right) \nabla_{\perp} \partial_{x}^{2} h+\frac{\kappa}{\eta}\left(\frac{z^{2}}{2}-h z\right) \nabla_{\perp} \nabla_{\perp}^{4} h- \\
& -\frac{\hat{C}}{\eta}\left(\frac{z^{2}}{2}-h z\right) \nabla_{\perp} \partial_{y} \theta+\frac{\Delta \mu}{\eta}\left(\frac{z^{2}}{2}-h z\right)\left[\left(\partial_{x} c+c_{0} \partial_{y} \theta+c_{0} h^{-1} \partial_{x} h\right) \hat{\mathbf{e}_{\mathbf{x}}}+c_{0} \partial_{x} \theta \hat{\mathbf{e}_{\mathbf{y}}}\right] .
\end{aligned}
$$

Equation of motion of $h$ may be obtained by using the incompressibility of the velocity (just as before). We obtain

$$
\begin{aligned}
\frac{\partial h}{\partial t}= & \frac{h_{0}^{2}}{\eta}\left[\frac{5 \Delta \mu h_{0}^{2} c_{0}}{16} q^{2} q_{x}^{2} h-\frac{3 c_{0} \Delta \mu}{4} q_{x}^{2} h-\frac{4 \kappa h_{0}}{3} q^{6} h+i \frac{\hat{C} h_{0}}{3} q^{2} q_{y} \theta\right. \\
& \left.-1 / 3\left(\Delta \mu h_{0} q_{x}^{2} c+2 \Delta \mu c_{0} h_{0} q_{x} q_{y} \theta+\Delta \mu c_{0} q_{x}^{2} h\right)\right]
\end{aligned}
$$

The equation motion for the polar orientation field is the same as for Model I above. After $z$-averaging one obtains the final explicit form given by Eq. (36) as given in Sec. VI The concentration field obeys the same equation (14) as for Model I.

\section{DYNAMICS AND INSTABILITIES}

\section{A. Results from Model I}

Having set up the effective $2 d$ coupled equations of motion for $h(\mathbf{q}, t), \theta(\mathbf{q}, t)$ and $c(\mathbf{q}, t)$, we now examine the linear instabilities about a uniform state $h=0, \theta=0$ and $c=0$. We begin with the extreme case with immotile but active polar particles, i.e., $v_{0}=0$ but $\Delta \mu \neq 0$. Clearly, in this limit, concentration $c$ decouples from $h$ and $\theta$. We analyse the mode structure in the low q limit. Notable characters of the mode structures are

- There are $O\left(q^{0}\right)$ anisotropic contributions proportional to $\Delta \mu c_{0} / \eta$, which are of purely active origin. In detail: Assuming the fields to have time-dependence of the form $\exp (\Lambda t)$, we have for the eigenvalues $\Lambda$ of the linear stability matrix

$$
\begin{aligned}
\Lambda\left(q_{x}, q_{y}\right)= & \frac{(\lambda-1) \Delta \mu c_{0} q_{y}^{2}}{4 \eta q^{2}}+\frac{(\lambda+1) \Delta \mu c_{0} q_{x}^{2}}{4 \eta q^{2}}-\frac{\Delta \mu c_{0} q_{x}^{2}}{\eta q^{2}} \pm \frac{1}{2}\left[\left\{\frac{(\lambda-1) \Delta \mu c_{0} q_{y}^{2}}{2 \eta q^{2}}\right.\right. \\
& \left.\left.+\frac{(\lambda+1) \Delta \mu c_{0} q_{x}^{2}}{2 \eta q^{2}}-\frac{2 \Delta \mu c_{0} q_{x}^{2}}{\eta q^{2}}\right\}^{2}-4 \frac{(\Delta \mu)^{2} c_{0}^{2} q_{x}^{2}}{\eta^{2} q^{4}}\left\{(\lambda-1) q_{y}^{2}-(\lambda+1) q_{x}^{2}\right\}\right]^{\frac{1}{2}}
\end{aligned}
$$


Eigenvalues are anisotropic (in the Fourier space) and have complicated dependences on $q_{x}$ and $q_{y}$. It may however be noted that when $2 q_{x}^{2}-(\lambda-1) q_{y}^{2} / 2-(\lambda+1) q_{x}^{2} / 2$ have a definite signature (either positive of negative) and $(\lambda-1) q_{y}^{2}-(\lambda+1) q_{x}^{2}$ small, one may expand the square root in (20) binomially. One of the solutions of $\Lambda$ is then proportional to $\Delta \mu\left[(\lambda-1) q_{y}^{2}-(\lambda+1) q_{x}^{2}\right] /\left[2 q_{x}^{2}-(\lambda-1) q_{y}^{2} / 2-(\lambda+1) q_{x}^{2} / 2\right]$ to the leading order in smallness, and is thus unstable just above or below the line $(\lambda-1) q_{y}^{2}=(\lambda+1) q_{x}^{2}$, depending upon the signature of $\Delta \mu$. This is clearly indicative of instabilities for either sign of $\Delta \mu$. The unstable eigenvalue gets a particularly simpler form for large $|\lambda| \gg 1$. We find one of the eigenvalues

$$
\Lambda\left(q_{x}, q_{y}\right) \sim \frac{\Delta \mu c_{0} q_{x}^{2}}{\eta q^{4}}\left(q_{y}^{2}-q_{x}^{2}\right)
$$

displaying clearly the instability for either signature of $\Delta \mu$ clearly. Further, for $q_{x}=0$ and $q_{y}=0$ one may separately obtain the eigenfrequencies $\Lambda$ as

$$
\begin{array}{r}
\Lambda\left(q_{x}=0, q_{y}\right)=\frac{\lambda-1}{2 \eta} \Delta \mu c_{0}, 0, \\
\Lambda\left(q_{x}, q_{y}=0\right)=-\frac{2 \Delta \mu c_{0}}{\eta}, \frac{\lambda+1}{2 \eta} \Delta \mu c_{0} .
\end{array}
$$

Notice that the mathematical origin for the instability for either signature of $\Delta \mu$ lies essentially in the mixed or cross-coupling terms (i.e., the $h$-dependent term in the $\theta$-equation and viceversa) and the fact that, for the leading order in wavevector $q$, the terms on the right hand side of Eq. (12) have the same sign; similarly the terms on the right hand side of Eq. (13) have the same sign. Let us try to see why this is so: There is only one source of activity in the model, viz, the active stress given by Eq. (4). Now, this contributes to two different quantities in the effective $2 d$ descriptions: (i) $2 d$ active pressure, that depends on $h$ and (ii) $2 d$ analogue of the bulk $3 d$ active shear stress that depends upon $\theta$. The dynamic of $h$ and $\theta$ depend upon both of them. This explains the presence of the cross-coupling terms in the dynamics. Because of the general structure of the Stokes Eq. and our approximation of small $v_{z}$ (lubrication approximation), the $2 d$ active pressure and the $2 d$ shear stress come with the same signatures, the feature that is responsible for the occurrence of the instabilities mentioned here [39]. Since physical fields $h(\mathbf{r})$ and $\theta(\mathbf{r})$ depends upon the Fourier modes $h(\mathbf{q})$ and $\theta(\mathbf{q})$ for all $\mathbf{q}, h(\mathbf{r})$ and $\theta(\mathbf{r})$ display instability for both signs of $\Delta \mu$ at short enough wavenumbers. Note that these $q$-independent contributions do not mean the modes have finite life time at vanishing $\mathbf{q}$; such $q$-independent behaviour is nothing but a consequence of using the Stokes' equation (instead of the Navier-Stokes equation) for the velocity field. In the Stokes' approximation one neglects the inertia in comparison with the viscous term. Of course, for very low wavenumber this is no longer valid and inertia effects will be important. Thus the above expressions of $\Lambda$ cannot be used in the limit $\mathbf{q} \rightarrow 0$. Note, however, that this does not question the mathematical validity or consistency of the Stokesian hydrodynamics that we have used. At the linear level, all modes labeled by $\mathbf{q}$ are decoupled. Hence, the properties of one particular mode is unaffected by any other mode with any wavevector (including vanishing wavevectors). Since the initial amplitudes of the (small) perturbations considered here can be taken to zero smoothly, the validity of the Stokesian hydrodynamics with linearised approximation remains sound.

- At the $O\left(q^{2}\right)$ the contributions are easily presentable in the limits $q_{x}=0$ and $q_{y}=0$, when their expressions simplify considerably. the contributions are still purely real and display instability just like the contributions at $q^{0}$. Parts of the contributions are active, parts are however from the equilibrium part of the dynamics. We find

$$
\begin{aligned}
& \Lambda\left(q_{x}=0, q_{y}\right)=\frac{\lambda-1}{2 \eta} \Delta \mu c_{0}-D q_{y}^{2}, 0, \\
& \Lambda\left(q_{x}, q_{y}=0\right)=-\frac{2 \Delta \mu c_{0}}{\eta}+\frac{\Delta \mu c_{0} h_{0}^{2}}{4 \eta} q_{x}^{2}, \frac{\lambda+1}{2 \eta} \Delta \mu c_{0}-D q_{x}^{2} .
\end{aligned}
$$

A schematic diagram of the eigenvalues is given in Fig. 3. We thus see that there are both stable (coming from $D>0$ ) and unstable active contributions at $O\left(q^{2}\right)$. Thus depending upon the numerical value of $\Delta \mu$, there may be instabilities at $O\left(q^{2}\right)$. Ignoring anisotropy, Eq. (24) or (25) allows identify a length scale

$$
L_{c}=\sqrt{\frac{D \eta}{c_{0} \Delta \mu}},
$$

such that systems with linear dimensions $L>L_{c}$ are unstable. Note: Due to the anisotropy, the length scale $L_{c}$ should depend upon the polar angle which may be obtained in a more detailed calculation. Propagating 

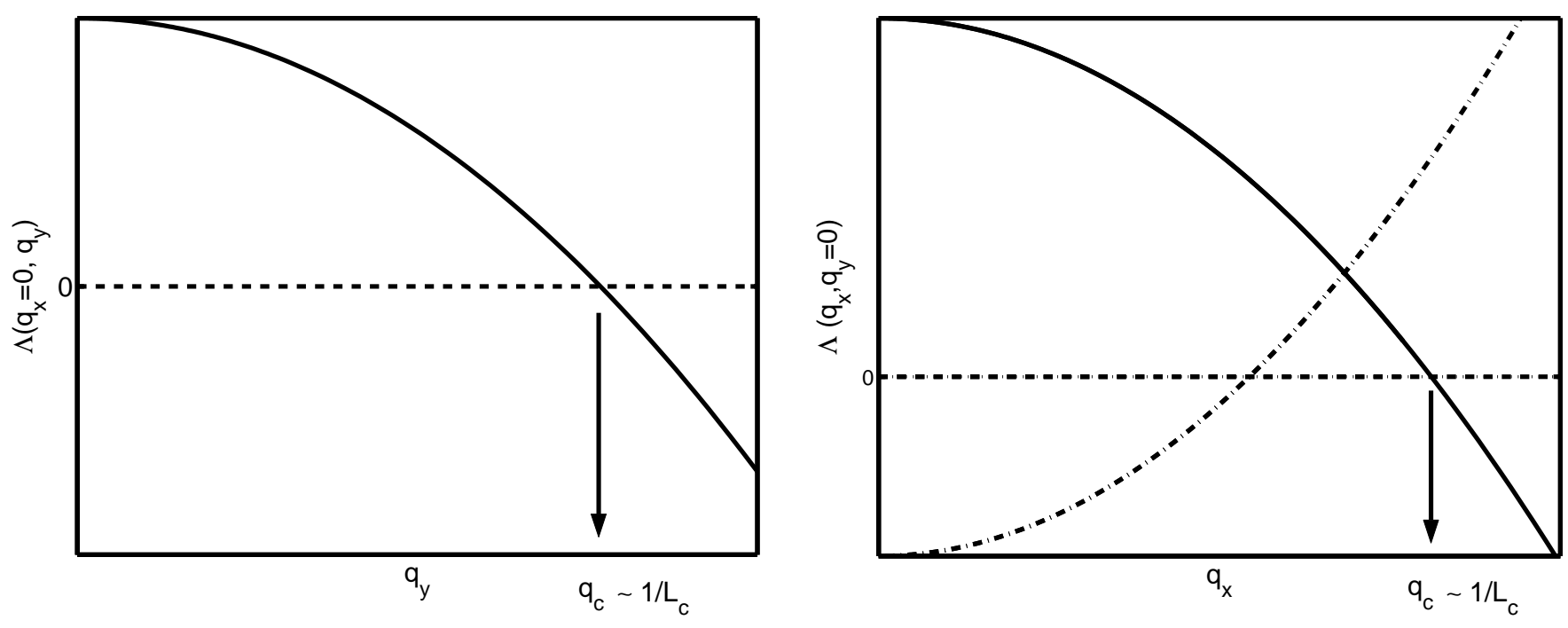

FIG. 3: Schematic plots showing the eigenvalues $\Lambda$ in the two limits up to $O\left(q^{2}\right)$

for Model I for $\Delta \mu>0$ - left: $\Lambda\left(q_{x}=0, q_{y}\right)$ vs $q_{y}$ (a downward parabola, continuous line), right: $\Lambda\left(q_{x}, q_{y}=0\right)$ vs $q_{x}$ (upward (dot-dash) and downward (continuous line) parabolas). The broken horizontal line in both the plots denote $\Lambda=0$; its meeting point with the downward parabola yields $q_{c} \sim 1 / L_{c}$.

modes due to the tilt-curvature coupling appear at even higher $q$, at $O\left(q^{5 / 2}\right)$. These propagating modes are strongly damped at smallest wavenumbers. Finally, at a sufficiently large $q$, all the instabilities are cut-off by the bending stiffness $\kappa$ of the membrane.

What could be a typical value for $L_{c}$ ? We take linear size of an active particle $\sim 1 \mathrm{~nm}$, volume fraction $\phi=c_{0} a^{3}=1$ (since we assume to be in an ordered state), $D \eta \sim K \sim 10^{-6}$ dyne $/ \mathrm{cm}$ where $K$ is a Frank elastic constant. Estimation of $\Delta \mu$ is more ambiguous: We use the fact that approximately $7 \mathrm{KCal}$ energy released per mole of ATP due to its hydrolysis. Since 1 molar mass of ATP $\sim 500$, we obtain from its definition $\Delta \mu \sim 7 \mathrm{KCal} /\left(500 \mathrm{gm} / 10^{23}\right)$ the free energy release per unit mass per molecule. All these, however, lead to $L_{c} \sim 10 \mathrm{~nm}$, a value rather low compared to the linear dimensions of an eukaryotic cell. However, we have ignored anisotropy while estimating $L_{c}$. Moreover, for a realistic situation the effects of the ambient fluid is likely to be significant and should affect $L_{c}$. In any case, a direct comparison with a living cell is not very effective due to the simplifying approximations and the assumption of an ordered state of the active particles that we have made.

If we now consider motile particles $\left(v_{0} \neq 0\right)$ but continue to ignore $c$, the most dominant effect is that one of the modes will now pick up a propagating part with speed $v_{0}$ and dispersion proportional to $q_{x}$. Although this is still subdominant to the most leading order instabilities, for sufficiently large $v_{0}$, the propagating modes will be observed and the instabilities at higher $q$ corresponding to this particular mode will now be moving. Finally, when the dynamics of $c$ is considered, the eigenmodes have complex forms as a function of $\mathbf{q}$. A simple way to analyse them is to look at special limits of either $q_{x}=0$ or $q_{y}=0$ (an alternative way, considered in Ref. [29], is to examine the limit $\left.\Gamma \hat{C} / v_{0} \gg 1\right)$. We find

$$
\begin{aligned}
\Lambda\left(q_{x}=0, q_{y}\right)= & \frac{(\lambda-1)}{2 \eta} \Delta \mu c_{0}-D q_{y}^{2} \pm \frac{1}{2}\left[\left\{D q_{y}^{2}-\frac{(\lambda-1)}{2 \eta} \Delta \mu c_{0}\right\}^{2}\right. \\
& \left.-4 v_{0} c_{0} \xi q_{y}^{2}\right]^{1 / 2}, \quad-\frac{\kappa h_{0}}{\eta} q_{y}^{4} \\
\Lambda\left(q_{x}, q_{y}=0\right)= & \frac{2 \Delta \mu c_{0} h_{0}^{2}}{4 \eta} q_{x}^{2}-\frac{\kappa h_{0}}{\eta} q_{x}^{4}-\frac{2 \Delta \mu c_{0}}{\eta}, \\
& (\lambda+1) \frac{\Delta \mu c_{0}}{2 \eta}-D q_{x}^{2}-i a_{1} v_{0} q_{x}, \quad-i v_{0} q_{x} .
\end{aligned}
$$

Thus again in the intermediate wavenumber range there are $O\left(q^{2}\right)$ instabilities for sufficiently large $\Delta \mu$. In addition, there are propagating waves coming from the concentration-orientation coupling. Thus the instabilities and the resulting patterns will be moving. Finally, at even larger wavenumber, all eigenmodes are stable owing to the bending stiffness of the membrane. If $\Delta \mu=0$, the system is stable which is expected in the equilibrium limit. In Fig. 4 we 
pictorially show the presence of instability for both signs of $\Delta \mu$ in plots of $\frac{\Delta \mu c_{0}}{\eta}$ vs $D$ and $\Delta \mu c_{0}$ vs $\kappa$ for eigenvalue $\Lambda\left(q_{x}, q_{y}=0\right)$ for a given value of $q_{x}$. The line in the $D-\Delta \mu c_{0} / \eta$ plane are given by $D q_{x}^{2}=(\lambda+1) \Delta \mu c_{0} / 2 \eta$ for a given $q_{x}$ and the line in the $\kappa-\Delta \mu c_{0} / \eta$ plane is given by $\kappa h_{0} q_{x}^{4}=-2 \Delta \mu c_{0}+\Delta \mu c_{0} h_{0}^{2} q_{x}^{2} / 2$ again for a given $q_{x}$. The plots clearly indicate that for any of the signatures of $\Delta \mu$ some regions in either of the plots display instability.
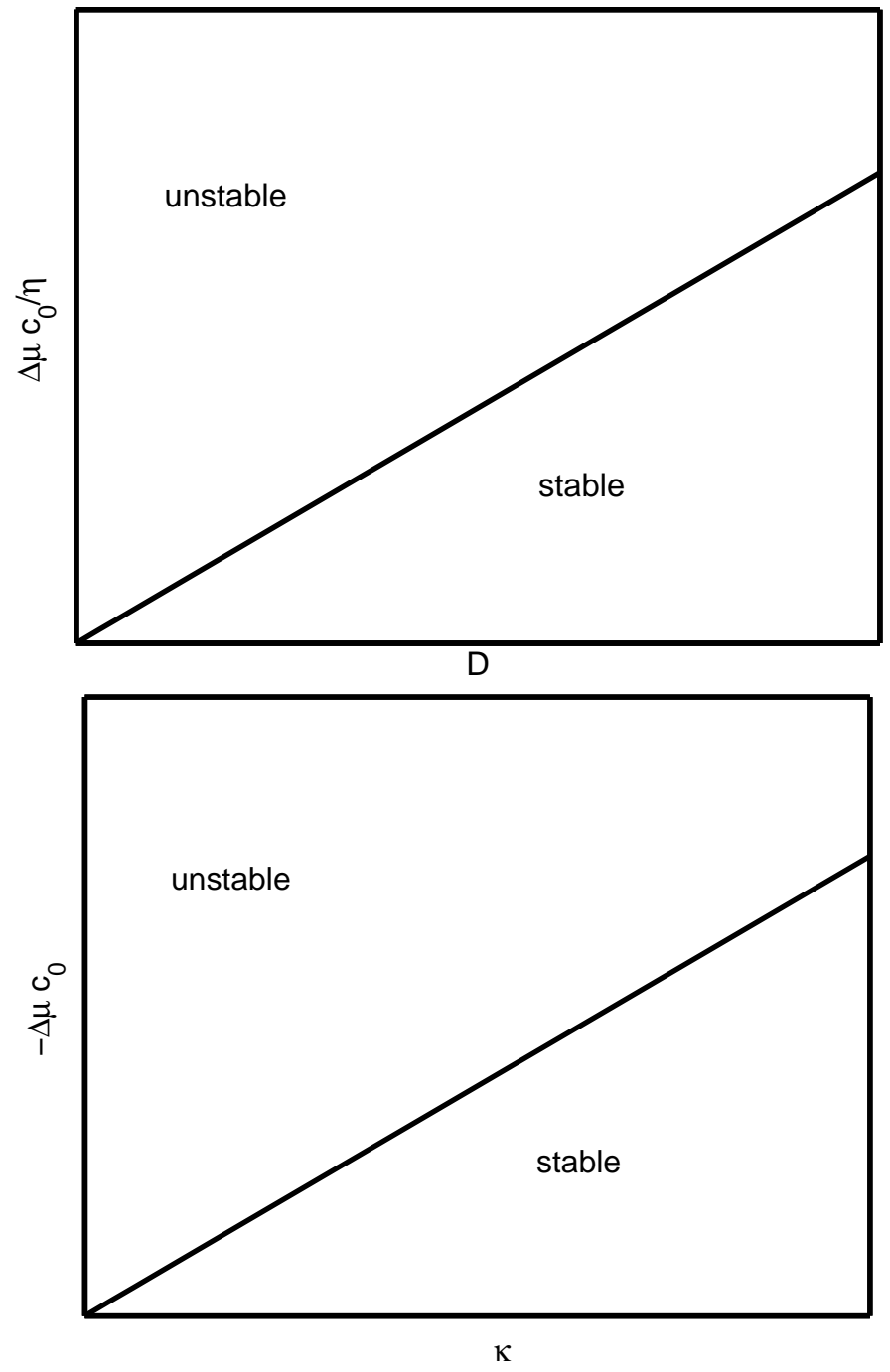

FIG. 4: A schematic diagram showing the unstable and stable regions in a phase space diagram for Model I with a given $q_{x}$ (left: $\frac{\Delta \mu c_{0}}{\eta}$ vs $D$, right: $\Delta \mu c_{0}$ vs $\left.\kappa\right)$ for eigenvalue $\Lambda\left(q_{x}, q_{y}=0\right)$. The lines are given by the zeros of $\Lambda\left(q_{x}, q_{y}=0\right)=0$ for a given $q_{x}$, ignoring $v_{0}$.

\section{B. Results from Model II}

When there is a solid substrate, the long wavelength dynamics is same as that in Ref. [29]. We present them here for direct comparison with the free standing case. The two eigenmodes $\Lambda$ of the linearised coupled dynamical equations 
of $h$ and $\theta$ have the form (setting $v_{0}=0$ )

$$
\begin{aligned}
\Lambda= & -\frac{11 \Delta \mu c_{0} h_{0}^{2}}{24 \eta} q_{x}^{2}-\frac{\Delta \mu c_{0} h_{0}^{2}}{12 \eta} q_{y}^{2}-\frac{D q^{2}}{2}+\frac{\lambda \Delta \mu c_{0} h_{0}^{2}}{6 \eta} q^{2} \\
& \pm \frac{1}{2}\left[\left\{-\frac{11 \Delta \mu c_{0} h_{0}^{2}}{12 \eta} q_{x}^{2}-\frac{\Delta \mu c_{0} h_{0}^{2}}{6 \eta} q_{y}^{2}-D q^{2}+\frac{\lambda \Delta \mu c_{0} h_{0}^{2}}{3 \eta} q^{2}\right\}^{2}-8 i \hat{C} \frac{\Delta \mu c_{0} h_{0}^{3}}{3 \eta} q_{x} q_{y}^{2}-\frac{4 \Gamma \hat{C} h_{0}^{3}}{3 \eta} q_{y}^{2} q^{2}\right. \\
& \left.-\frac{13 \Delta \mu c_{0} D h_{0}^{2}}{3 \eta} q_{x}^{2} q^{2}+\frac{13(\Delta \mu)^{2} c_{0}^{2} h_{0}^{4}}{18 \eta^{2}} q_{x}^{4}-\frac{13(\Delta \mu)^{2} c_{0}^{2} h_{0}^{4}}{18 \eta^{2}} q_{x}^{2} q_{y}^{2}+\frac{13(\Delta \mu)^{2} c_{0}^{2} h_{0}^{4}}{9 \eta^{2}} q_{x}^{2} q^{2}\right]^{1 / 2}
\end{aligned}
$$

As in Ref. [29], to the lowest order in wavevector, there is a traveling instability with a growth rate proportional to $q_{y} q_{x}^{1 / 2}$; the mode displaying an instability travels in the $+x$ direction (-x direction) if $\Delta \mu>0(\Delta \mu<0)$. If we now include $v_{0}$, but still ignore the dynamics of $c$, the eigenfrequencies in the limits $q_{x}=0$ and $q_{y}=0$ are

$$
\begin{aligned}
\Lambda\left(q_{x}, q_{y}=0\right)= & -\frac{13 \Delta \mu c_{0} h_{0}^{2}}{12 \eta} q_{x}^{2}+\frac{5 \Delta \mu c_{0} h_{0}^{4} q_{x}^{4}}{16 \eta}-\frac{4 \kappa h_{0}^{3}}{3 \eta} q_{x}^{6}, \\
& -i a_{1} v_{0} q_{x}-D q_{x}^{2}+(2 \lambda+1) \frac{\Delta \mu c_{0} h_{0}^{2}}{6 \eta} q_{x}^{2}, \\
\Lambda\left(q_{x}=0, q_{y}\right)= & -\frac{D q_{y}^{2}}{2}+(2 \lambda-1) \frac{\Delta \mu c_{0} h_{0}^{2}}{12 \eta} q_{y}^{2} \pm \frac{1}{2}\left[\left\{D q_{y}^{2}-(2 \lambda-1) \frac{\Delta \mu c_{0} h_{0}^{2}}{6 \eta} q_{y}^{2}\right\}^{2}-\frac{4 \hat{C}^{2} h_{0}^{3}}{3 \eta} q_{y}^{4}\right]^{\frac{1}{2}} .
\end{aligned}
$$

It is worth pointing out the basic differences between the eigenvalues for a free standing system given by (24)25) and those for a system with a solid substrate below given by (29) or those in Ref. [29], whose calculational framework has been largely used here. In the former case, eigenvalues are $q$-independent for small $\mathbf{q}$. This is a consequence of the long-ranged hydrodynamic interactions. In contrast, when there is a solid substrate, the eigenvalues smoothly tend to zero as $q \rightarrow 0$. This is because any long-ranged hydrodynamic interactions are screened by the solid substrate below. This means, no matter how small the activity $(\Delta \mu)$ is, for a large enough system (with linear dimension $L>L_{c}$ ) the system in Model I gets unstable. In contrast, for model II the screening of the hydrodynamic interactions yields that as long as the system has a thickness smaller than the critical thickness $h_{0 c} \sim \sqrt{\frac{D \eta}{c_{0} \Delta \mu}}$ for a given $\Delta \mu$, the system with arbitrarily large in-plane linear dimensions remains stable. This crucial difference apart, eigenvalues (30131) show instabilities for sufficiently high $\Delta \mu$. Although the latter features are similar to that for a free standing system (Model I), the underlying physical mechanisms are different: Unlike Model I, where hydrodynamic interactions are responsible for type of the instabilities observed there, for Model II, the tilt-concentration coupling characterised by the coupling constant $\hat{C}[29]$ is responsible.

Regardless of the details of the boundary conditions on the system, qualitative implications of both hold for a variety of phenomenologies. First of all, since $v_{x}$ and $v_{y}$ depend upon $h, \theta$ and $c$, as given in Eqs. (11) and (10) for Model I, and Eq. (18) for Model II, as soon as one of the modes become unstable, the initial homogeneous non-flowing state will be unstable and the active fluid starts to flow. While our simple model calculations by themselves cannot capture the full phenomenologies of flows in cell cortex (see, e.g., Refs. 24, 25]; see also Ref. 26] for a $1 d$ model for pattern formation in active fluids), our results open up possibilities of flows in the cell cortex within calculations in a simple setting. We show instabilities develop under generic conditions. In addition, there may be waves under various conditions. Our results are universal in the sense that they do not refer to any specific cell or do not depend upon very specific biochemical processes. Although our system and the results which follow cannot be directly related to any biological systems due to our several simplifying approximations, we believe our results will inspire more realistic studies on (moving or static) patterns ultimately formed in cell membranes (see, e.g., Refs. 28, 40, 41]). Finally, in view of our results here, let us briefly consider the possible structure of the effective $2 d$ model with a polar order that is perpendicular to the surfaces at $z=0$ and $h$. While quantitative predictions require detailed calculations, we can already make the following observations, based upon the framework developed above. In this case, again assuming a flat bottom surface (either a solid substrate or a free active fluid surface with a very large surface tension) the boundary conditions on the polarisation $\mathbf{p}$ are $p_{z}=1$ at $z=0$ and $\mathbf{p} \cdot \boldsymbol{\nabla} h=1$ at $z=h$. For small fluctuations of $p_{x}$ and $p_{y}$ (fluctuations in $p_{z}$ will be second order in smallness) the boundary condition at $z=h$ implies $p_{x}=\partial_{x} h / 2, p_{y}=\partial_{y} h / 2$. Further, $p_{x}$ and $p_{y}$ are constrained to be zero at $z=0$. Thus, in a $z$-averaged description as above, $p_{x}$ and $p_{y}$ are slaved to $\boldsymbol{\nabla} h$. Hence, staying within the framework of $2 d$ effective description as developed above, the effective dynamics will be described by $2 d$ equations of motion of height $h$ and concentration $c$ only. This effective dynamics is now expected to display signatures of instabilities and a spontaneous flow transition akin to the 
Frederiks transition of equilibrium nematic liquid crystals at a critical thickness for contractile active stress with a given value of $\Delta \mu$, similar to those discussed in Refs. [48, 49]. Details of these will be discussed elsewhere.

\section{DIFFUSION COEFFICIENTS}

The in-plane diffusive motion of membrane bound protein are a key ingredient in a great many biological functions including exchange of information, material and/or energy. A complete picture of how cells function and interact with their immediate surroundings requires understanding of this diffusive phenomena. The recent progress in experimental techniques to measure lateral $\left(D_{L}\right)$ and rotational $\left(D_{R}\right)$ diffusion coefficients, like fluorescence correlation spectroscopy 42], single particle tracking [43], or fluorescence recovery after photobleaching [44], has revealed that many of the functions performed by proteins are crucially influenced by the diffusive behavior of the proteins [45]. Apart from its obvious biological significance, diffusion of membrane-bound proteins is a good example of $2 d$ diffusion in a fluctuation background (here the membrane), where the fluctuations are of nonequilibrium origin. Calculation of $D_{L}$ in a strictly $2 d$ flow is subtle due to the divergence associated with the solutions of the $2 d$ Stokes' equation, known as the Stokes' paradox in the literature [46]. In order to overcome this Stokes' paradox, Saffman and Delbrück 31] considered the mobility of a very thin, rigid object in a narrow almost $2 d$ perfectly flat fluid layer that is surrounded on both sides by a further liquid and obtained finite results for $D_{L}$. Calculation of $D_{R}$ does not suffer from any such subtle issues. Recently in Ref. [47] the authors calculated $D_{L}$ for a protein molecule in a bare membrane. In none of these theoretical examples, the nonequilibrium nature of the membrane dynamics and the associated active cortical actin layer have been considered, although the cellular cytoskeleton is often linked with the mobility of a protein molecule in the membrane, see, e.g., Refs. 7]. We here elucidate possible effects of the cortical actin layer nonequilibrium fluctuations on the measured value of the diffusion coefficients within our effective $2 d$ coarse-grained model. As we shall show below, the ensuing algebraic details is rather complicated. Hence we confine ourselves here to a treatment at the scaling level only. This suffices for our purposes here.

Let us begin with the formal definition of the diffusion coefficients: Since we have an anisotropic system, we consider the general lateral diffusion tensor $D_{i j}^{L}$ which is defined by the relation $\left\langle r_{i}(t) r_{j}(t)\right\rangle=2 D_{i j}^{L} t$, where $r_{i}(t)$ is the Lagrangian coordinate of the protein molecule in the membrane at time $t$. Equating the Lagrangian velocity of the particle with the local in-plane $2 d$ velocity field, away from the instability threshold the above definition leads to $D_{i j}^{L}=\frac{1}{2} \int \frac{d^{2} q}{(2 \pi)^{2}}\left\langle v_{i}(\mathbf{q}, \omega=0) v_{j}(-\mathbf{q}, \omega=0)\right\rangle$. We calculate the two lateral diffusion coefficients $D_{x x}^{L}$ and $D_{y y}^{L}$ below. Similar to the lateral diffusion coefficients, the rotational diffusion coefficient $D_{R}$ is given by $D_{R}=\frac{1}{2} \int \frac{d^{2} q}{(2 \pi)^{2}}\langle| \Omega(\mathbf{q}, \omega=$ $\left.0)\left.\right|^{2}\right\rangle$, where $\Omega$ is the $z$-component of the vorticity tensor defined above. Thus enumeration of diffusion coefficients requires calculations of certain velocity correlation functions which may be done by stochastically driving the $2 d$ equations of motion developed above. In order to simplify our calculations we ignore any local effect of the protein molecule on $\kappa$ [47]. Further, we set $v_{0}=0$, thus concentration $c$ decouples from the dynamics at the lowest order in q. We present the main results below, with some of the algebraic details available in Appendix II. For a free standing system, we add zero-mean conserved Gaussian distributed thermal noises in Eqs. (10) [11) and a zero-mean thermal Gaussian distributed white noise in Eq. (35). Finally, we calculate the diffusion coefficients for immotile active particles, i.e., $v_{0}=0$. In addition, we ignore the concentration field $c$ in the following calculations.

We consider Model I first. Calculations are considerably simplified if we consider the dynamics only at the long wavelength limit, which suffices for our purposes here. In that limit, correlators of $h, \theta, v_{x}, v_{y}$ are available in Appendix II. The noticeable feature is that all of Eqs. (41) have parts which diverge beyond a critical system size $L_{c}$. Ignoring anisotropy, the length scale $L_{c}$ is given by Eq. (26) above. From the correlators (41) in Appendix II. we obtain for the lateral diffusion coefficients (we show only the diverging parts below)

$$
\begin{aligned}
& D_{x x} \sim \frac{K_{B} T}{h_{0}} \int \frac{d^{2} q}{(2 \pi)^{2}} \frac{\left(\frac{3 \Delta \mu q_{y} c_{0}}{\eta q^{2}}\right)^{2}}{\left[D q^{2}+\frac{(\lambda-1) c_{0} \Delta \mu}{\eta q^{2}} q_{y}^{2}-\frac{(\lambda+1) c_{0} \Delta \mu}{2 \eta q^{2}} q_{x}^{2}\right]^{2}} \frac{1}{\eta}\left[1+\left(\frac{q_{y}(\lambda-1)}{2 q_{x}}\right)\right] \\
& D_{y y} \sim \frac{K_{B} T}{h_{0}} \int \frac{d^{2} q}{(2 \pi)^{2}} \frac{\left(\frac{\Delta \mu q_{x} c_{0}}{\eta q^{2}}\right)^{2}}{\left[D q^{2}+\frac{(\lambda-1) c_{0} \Delta \mu}{\eta q^{2}} q_{y}^{2}-\frac{(\lambda+1) c_{0} \Delta \mu}{2 \eta q^{2}} q_{x}^{2}\right]^{2}} \frac{1}{\eta}\left[1+\left(\frac{q_{y}(\lambda-1)}{2 q_{x}}\right)\right]
\end{aligned}
$$

Due to the complicated nature of the expressions (32) we do not attempt to evaluate them exactly. Instead, we treat them at the scaling level. For a free standing system without activity, $D_{x x}$ and $D_{y y}$ depend on the system size $L$ logarithmically, i.e., as $\log L$, which is the equilibrium contribution. This contribution survives even when $\Delta \mu \neq 0$ which we do not show explicitly above. Evidently, for an active system, the dependence of the active contributions 
to $D_{x x}$ and $D_{y y}$ on $L$ are very different, as given by Eq. (26). Thus at the scaling level we obtain

$$
D_{x x}, D_{y y} \sim \frac{K_{B} T}{h_{0} \eta} L_{c}^{4}\left(L_{c}^{2}-L^{2}\right)^{-2} .
$$

for $L \ll L_{c}$. Thus as $L$ increases, $D_{x x}$ and $D_{y y}$ rise with $L$ in power law fashion, unlike in equilibrium systems where such rises are logarithmic in $L$. Similarly, $D_{R}$, for a membrane on a thin non-active fluid film (i.e., in thermal equilibrium), has no dependence on $L$, it instead depends on the small-scale cut-off $l$ (of the order of the particle size) as $l^{-2}$. In contrast, when the fluid film is active, $D_{R} \sim \frac{K_{B} T}{\eta h_{0}} \frac{L_{c}^{2}}{\left(L_{c}^{2}-L^{2}\right)^{2}}$ below the threshold. Thus, similar to $D_{x x}^{L}$ and $D_{y y}^{L}, D_{R}^{L}$ increases as $L$ increases. For a thin inflexible isotropic fluid layer with viscosity $\eta$ surrounded by a bulk fluid of viscosity $\eta_{1}$ with $\eta_{1} / \eta$ finite, theoretical calculations of Ref. [31] diffusion coefficient of an inclusion of a small but finite size is finite. Our work is thus an extension of Ref. 31], incorporating effects of orientation degrees of freedom and a fluid membrane, but staying at the special simple limit of $\eta_{1} / \eta \rightarrow 0$. In addition, our assumption of inflexible active fluid-isotropic bulk fluid interface will not strictly hold in a real cell. Thus more refined calculations are needed for better quantative estimations. We conclude this Section by mentioning that there are two important physical effects which we have not considered. First of all, it is well-known that for a membrane with a finite thickness in thermal equilibrium, fluctuations increase the effective thickness [14] which in turn reduces the diffusivity. Similar effects should be observed in the present problem as well, whose quantitative enumeration requires further work which we do not discuss here. However, our results above holds in the limit of zero membrane thickness (the parameter $h_{0}$ here corresponds to the average thickness of the active fluid layer, and not of the thickness of the membrane at the top). Secondly, in view of the findings of Ref. [15] that a quenched rough surface reduces the effective diffusion coefficient substantially, whereas, for an annealed surface the reduction is relatively small, it would be important to investigate the analogous effects in the present problem. However, direct application of the results of Ref. 15] to our problem is difficult due to the presence of additional degrees of freedom (polarisation fluctuation $\theta$ ) with long-ranged correlations and the active stress. Further work is necessary to settle this issue properly. This is beyond the scope of the present work.

In order to calculate diffusion coefficients of an inclusion for Model II we need to set $\hat{c}=0$, since otherwise there are underdamped propagating modes in the system. From the correlators (43) we find that, unlike the free standing system, there are no critical lateral size beyond which instabilities set in; further their dependence on $L$ is $\log L$. Instead, now there is a critical thickness $h_{0 c}$ such that for a film with thickness larger than $h_{0 c}, h_{0 c} \sim \sqrt{\frac{D \eta}{c_{0} \Delta \mu}}$ spontaneous flow instabilities akin to the Frederiks transition in equilibrium nematics set in [48, 49]. For a system with $h_{0}<h_{0 c}$ we find

$$
D_{x x}^{s}, D_{y y}^{s} \sim \frac{K_{B} T}{\eta h_{0}} \frac{h_{0 c}^{4}}{\left(h_{0 c}^{2}-h_{0}^{2}\right)^{2}} \log L
$$

for $h_{0}$ smaller than the critical thickness $h_{0 c}$. Thus $D_{x x}^{s}$ and $D_{y y}^{s}$ depend explicitly on $h_{0}$, a measure of the system size. Note the differences between the expressions of diffusion coefficients in Model I and II. For Model I, they depend upon the linear size $L$ of the system in a way markedly different from the $\log L$ dependence as observed in equilibrium systems. In contrast, in Model II, they depend on $L$ as $\log L$; in addition however they acquire non-trivial dependences on $h_{0}$.

\section{SUMMARY AND OUTLOOK}

In this article we have set up $2 d$ coarse-grained equations for a coupled system of a fluid membrane and a thin layer of active cortical actins anchored to it in terms of a height field, orientation field and concentration of active particles. We considered two cases of a free standing system and a solid substrate under the system, separately. The distinguishing feature of the former case is the presence of long ranged hydrodynamic interactions. We discuss the generic instabilities and patterns which appear due to activity. We use our equations to calculate lateral and rotational diffusion coefficients of an inclusion in the membrane. For the case of a free standing film, we have assumed that the surface tension of the bottom free surface is large, so that fluctuations of that surface are suppressed and are not considered in the subsequent calculations. This is mainly a theoretically interesting case and for real experimentally testable systems, surface tension of the surfaces should be finite and hence the bottom surface will have fluctuations. Despite the limitations of our approximations, our results bring out the differences between the two cases (free standing system and system resting on a solid surface) very clearly. For the sake of analytical convenience we have ignored a few details of cell membranes, e.g., effects of the surface tension of the membrane, the role of active proteins in the membrane and local modulation of the bending stiffness due to the inclusion; these may however be important 
in a real biological set up. Qualitative features of our basic results should in principle be testable in standard cell biology experiments measuring, e.g., measurements of diffusion coefficients. However, direct comparisons with in vivo experiments will not be easy primarily due to our assumptions of idealised model and also due to many complicated features of a cell membrane (e.g., a cell membrane is actually a bilayer or the cortical actin is anchored to the membrane only at discrete junctions). Our work here relates to Ref. 31] in that we include new effects coming due to orientational degrees of freedom, active stress and finite membrane stiffness, but consider the limiting case where the ambient fluid viscosity is much smaller than the active fluid viscosity. Despite the limitations of our simplifying assumptions, out work shows the dramatic effects of hydrodynamic interactions and provides a first step towards more realistic calculations. We hope our work will stimulate more realistic calculations in this direction.

Apart from its phenomenological importance, we believe our work is a first step in formulating and understanding the $2 d$ coupled dynamics of a fluid membrane and driven orientational broken symmetry modes. It would be interesting to see how the predictions of Ref. [50] get modified due to the fluctuations of the membrane. Secondly the fluctuating orientational degrees of freedom should create an effective long ranged interactions (of nonequilibrium origin) between different parts of the membrane. It would be interesting to see whether such interactions may allow a finite temperature crumpling transition of a $2 d$ fluid membrane [51], something which is prohibited in equilibrium [35].

\section{APPENDIX I: FULL EQUATIONS FOR $\theta$}

In order to obtain an effective two-dimensional description of the dynamics of $\theta$ for a free standing system (Model I), we average over the $z$ direction to get

$$
\begin{aligned}
\frac{\partial \theta}{\partial t}= & -i a_{1} v_{o} q_{x} \theta-i \xi q_{y} c-D q^{2} \theta-\frac{\lambda}{\eta q^{2}}\left[\frac{3 \Delta \mu c_{0} h_{0}}{4} q_{y} q_{x}^{3} h+i \hat{C} q_{x} q^{4} \theta-\Gamma \kappa q_{x} q_{y} q^{4} h\right]-i \hat{C} q_{y} q^{2} h \\
& +\frac{(\lambda-1)}{2 \eta q^{2} h_{0}}\left[\Delta \mu h_{0} q_{x} q_{y} c+h_{0} c_{0} \Delta \mu q_{y}^{2} \theta+2 c_{0} \Delta \mu q_{x} q_{y} h\right]+\frac{(\lambda+1) c_{0} \Delta \mu}{2 \eta q^{2}} q_{x}^{2} \theta .
\end{aligned}
$$

The corresponding dynamical equation for $\theta$ when there is a solid substrate below (Model II) is

$$
\begin{aligned}
\partial_{t} \theta= & -i a_{1} v_{0} q_{x} \theta-i \xi q_{y} c+i \Gamma \hat{C} q_{y} h-D q^{2} \theta+\frac{7(\lambda-1)}{24 \eta} c_{0} \Delta \mu h_{0} q_{x} q_{y} h-\frac{\lambda}{16 \eta} \Delta \mu c_{0} h_{0}^{3} q_{y} q_{x}^{3} h \\
& +\frac{\lambda \kappa}{\eta} h_{0}^{2} q_{x} q_{y} q^{4} h-i \frac{\hat{C}}{3 \eta} h_{0}^{2} q_{y}^{2} q_{x} \theta+\frac{(\lambda-1)}{6 \eta} h_{0}^{2} \Delta \mu q_{x} q_{y} c+\frac{\lambda}{3 \eta} \Delta \mu c_{0} h_{0}^{2} q^{2} \theta-\frac{\Delta \mu}{6 \eta} c_{0} h_{0}^{2} q_{y}^{2} \theta \\
& +\frac{\Delta \mu}{6 \eta} c_{0} h_{0}^{2} q_{x}^{2} \theta+\frac{\lambda \sigma h_{0}^{2}}{3 \eta} q^{2} q_{x} q_{y} h .
\end{aligned}
$$

\section{APPENDIX II: CORRELATION FUNCTIONS}

\section{A. Model I: Free standing system}

Equations (11) and (10) reduce to

$$
\begin{aligned}
& v_{x}=-\frac{i}{\eta q^{2} h_{0}}\left[c_{0} h_{0} \Delta \mu q_{y} \theta+2 c_{0} \Delta \mu q_{x} h\right], \\
& v_{y}=-\frac{i}{\eta q^{2}} q_{x} \theta .
\end{aligned}
$$

The equations for $\theta$ and $h$ in the Fourier space in the long wavelength limit are

$$
\begin{aligned}
i \omega \theta+D q^{2} \theta-\frac{\lambda-1}{2 \eta q^{2} h_{0}}\left(h_{0} c_{0} \Delta \mu q_{y}^{2} \theta+2 c_{0} \Delta \mu q_{x} q_{y} h\right)-\frac{\lambda+1}{2 \eta q^{2}} c_{0} \Delta \mu q_{x}^{2} \theta & =g_{\theta}, \\
i \omega h-\frac{1}{\eta q^{2}}\left(2 c_{0} h_{0} \Delta \mu q_{x} q_{y} \theta+2 \Delta \mu c_{0} q_{x}^{2} h\right) & =g_{h},
\end{aligned}
$$

where $g_{\theta}$ are $g_{h}$ are zero-mean Gaussian distributed white and conserved noises respectively. In the above we have ignored the dynamics of $c$ and set $v_{0}=0$. Equations (40) and (40) may be solved in a straight forward way to obtain 
the corelators $\left\langle|h(\mathbf{q}, \omega)|^{2}\right\rangle$ and $\left\langle|\theta(\mathbf{q}, \omega)|^{2}\right\rangle$. The resulting expressions are lengthy and not very illuminating; we do not present the full expressions here. Instead, we obtain the correlators in the long wavelength limit and use them to obtain correlations functions $\left\langle\left|v_{x}(\mathbf{q}, \omega)\right|^{2}\right\rangle,\left\langle\left|v_{y}(\mathbf{q}, \omega)\right|^{2}\right\rangle$ and $\left\langle|\Omega(\mathbf{q}, \omega)|^{2}\right\rangle$ (where $\Omega(\mathbf{q})$ is the Fourier transform of the $z$-component of $\boldsymbol{\nabla} \times \mathbf{v}$ ). We find(ignoring parts which show no divergence)

$$
\begin{aligned}
\left\langle|h(\mathbf{q}, \omega=0)|^{2}\right\rangle & =2 K_{B} T / \eta \frac{\left(\frac{4 h_{0} q_{y}}{3 q_{x}}\right)^{2}\left[1+\left\{\frac{(\lambda-1) q_{y}}{q_{x}}\right\}^{2}\right]}{\left[D q^{2}+\frac{(\lambda-1) c_{0} \Delta \mu}{2 \eta q^{2}} q_{y}^{2}-\frac{(\lambda+1) c_{0} \Delta \mu}{2 \eta q^{2}} q_{x}^{2}\right]^{2}} \\
\left\langle|\theta(\mathbf{q}, \omega=0)|^{2}\right\rangle & =2 K_{B} T / \eta \frac{\left[1+\left\{\frac{(\lambda-1) q_{y}}{q_{x}}\right\}^{2}\right]}{\left[D q^{2}+\frac{(\lambda-1) c_{0} \Delta \mu}{2 \eta q^{2}} q_{y}^{2}-\frac{(\lambda+1) c_{0} \Delta \mu}{2 \eta q^{2}} q_{x}^{2}\right]^{2}}, \\
\left\langle\left|v_{x}(\mathbf{q}, \omega=0)\right|^{2}\right\rangle & =\left(\frac{3 \Delta \mu q_{y} c_{0}}{\eta q^{2}}\right)^{2} \frac{2 K_{B} T}{\eta} \frac{1+\left\{\frac{(\lambda-1) q_{y}}{q_{x}}\right\}^{2}}{\left[D q^{2}+\frac{(\lambda-1) c_{0} \Delta \mu}{2 \eta q^{2}} q_{y}^{2}-\frac{(\lambda+1) c_{0} \Delta \mu}{2 \eta q^{2}} q_{x}^{2}\right]^{2}}, \\
\left\langle\left|v_{y}(\mathbf{q}, \omega=0)\right|^{2}\right\rangle & =\left(\frac{c_{0} \Delta \mu q_{x}}{\eta q^{2}}\right)^{2} \frac{2 K_{B} T}{\eta} \frac{1+\left\{\frac{(\lambda-1) q_{y}}{q_{x}}\right\}^{2}}{\left[D q^{2}+\frac{(\lambda-1) c_{0} \Delta \mu}{2 \eta q^{2}} q_{y}^{2}-\frac{(\lambda+1) c_{0} \Delta \mu}{2 \eta q^{2}} q_{x}^{2}\right]^{2}}, \\
\left\langle|\Omega(\mathbf{q}, \omega=0)|^{2}\right\rangle & =\left(\frac{c_{0} \Delta \mu\left(q_{x}^{2}-3 q_{y}^{2}\right)}{\eta q^{2}}\right)^{2} \frac{2 K_{B} T}{\eta} \frac{1+\left\{\frac{(\lambda-1) q_{y}}{q_{x}}\right\}^{2}}{\left[D q^{2}+\frac{(\lambda-1) c_{0} \Delta \mu}{2 \eta q^{2}} q_{y}^{2}-\frac{(\lambda+1) c_{0} \Delta \mu}{2 \eta q^{2}} q_{x}^{2}\right]^{2}} .
\end{aligned}
$$

Evidently, all the correlators diverge beyond a typical critical system size $L_{c}$ defined above.

\section{B. System in contact with a solid substrate}

The stochastically driven $2 d$ equations of motion of $h, \theta, v_{x}$ and $v_{y}$ are

$$
\begin{aligned}
v_{x} & =-i \frac{c_{0} \Delta \mu h_{0}}{\eta} q_{x} h-i \frac{\Delta \mu c_{0} h_{0}^{2}}{2 \eta} q_{y} \theta+\xi_{x}^{s}, \\
v_{y} & =-i \frac{\Delta \mu c_{0} h_{0}^{2}}{2 \eta} q_{x} \theta+\xi_{y}^{s}, \\
\frac{\partial h}{\partial t} & =-\frac{13 c_{0} \Delta \mu h_{0}^{2} q_{x}^{2}}{12 \eta} h-\frac{2 h_{0}^{3} c_{0} \Delta \mu q_{x} q_{y}}{3 \eta} \theta+\xi_{h}^{s}, \\
\frac{\partial \theta}{\partial t} & =-D q^{2} \theta+\frac{7(\lambda-1) c_{0} h_{0} \Delta \mu q_{x} q_{y}}{24 \eta} h+\frac{\lambda c_{0} h_{0}^{2} \Delta \mu q^{2}}{3 \eta} \theta-\frac{\Delta \mu c_{0} h_{0}^{2} q_{y}^{2}}{6 \eta} \theta+\frac{\Delta \mu c_{0} h_{0}^{2} q_{x}^{2}}{6 \eta} \theta+\xi_{\theta}^{s} .
\end{aligned}
$$

Here the noises $\xi_{x}^{s}, \xi_{y}^{s}, \xi_{h}^{s}$ and $\xi_{\theta}^{s}$ are all zero-mean Gaussian white noises. Equations (42) lead to the velocity correlators

$$
\begin{aligned}
\left\langle\left|v_{x}(\mathbf{q}, \omega=0)\right|^{2}\right\rangle= & \left(\frac{9 \Delta \mu c_{0} h_{0}^{2} q_{y}}{78 \eta}\right)^{2} \frac{\left\langle\xi_{\theta}^{s}(\mathbf{q}, \omega=0) \xi_{\theta}^{s}(-\mathbf{q}, \omega=0)\right\rangle+\left\{\frac{7(\lambda-1) q_{y}}{26 h_{0} q_{x}}\right\}^{2}\left\langle\left|\xi_{h}^{s}(\mathbf{q}, \omega=0)\right|^{2}\right\rangle}{\left[D q^{2}-\frac{\lambda \Delta \mu c_{0} h_{0}^{2}}{3 \eta} q^{2}-\frac{\Delta \mu c_{0} h_{0}^{2}}{6 \eta} q_{x}^{2}+\frac{c_{0} \Delta \mu h_{0}^{2}}{\eta}\left\{\frac{7(\lambda-1)}{39}+\frac{1}{6}\right\} q_{y}^{2}\right]^{2}} \\
& +\left\langle\xi_{x}^{s}(\mathbf{q}, \omega=0) \xi_{x}^{s}(-\mathbf{q}, \omega=0)\right\rangle, \\
\left\langle\left|v_{y}(\mathbf{q}, \omega=0)\right|^{2}\right\rangle= & \left(\frac{\Delta \mu c_{0} h_{0}^{2} q_{x}}{2 \eta}\right)^{2} \frac{\left\langle\xi_{\theta}^{s}(\mathbf{q}, \omega=0) \xi_{\theta}^{s}(-\mathbf{q}, \omega=0)\right\rangle+\left\{\frac{7(\lambda-1) q_{y}}{26 h_{0} q_{x}}\right\}^{2}\left\langle\left|\xi_{h}^{s}(\mathbf{q}, \omega=0)\right|^{2}\right\rangle}{\left[D q^{2}-\frac{\lambda \Delta \mu c_{0} h_{0}^{2}}{3 \eta} q^{2}-\frac{\Delta \mu c_{0} h_{0}^{2}}{6 \eta} q_{x}^{2}+\frac{c_{0} \Delta \mu h_{0}^{2}}{\eta}\left\{\frac{7(\lambda-1)}{39}+\frac{1}{6}\right\} q_{y}^{2}\right]^{2}} \\
& +\left\langle\xi_{y}^{s}(\mathbf{q}, \omega) \xi_{y}^{s}(-\mathbf{q}, \omega=0)\right\rangle
\end{aligned}
$$

Thus there are no instabilities at any finite lateral dimension of the system, and hence there is no critical $L$, unlike the free standing system.

\section{ACKNOWLEDGEMENT}

The authors thank J.-F. Joanny and S. Ramaswamy for fruitful discussions and suggestions. AB gratefully acknowledges partial financial support in the form of the Max-Planck Partner Group at the Saha Institute of Nuclear Physics, 
Calcutta funded jointly by the Max-Planck- Gesellschaft (Germany) and the Department of Science and Technology (India) through the Partner Group programme (2009).

[1] U. Seifert, Adv. Phys., 46, 13 (1997).

[2] W. Helfrich, J. Phys. (France), 46, 1263 (1985); H.J. Deuling and W. Helfrich, ibid. 37, 1335 (1976).

[3] S. Ramaswamy, J. Prost, and T.C. Lubensky, Europhys. Lett., 27, 285 (1994); 23, 271 (1993).

[4] B. Alberts, D. Bray, J. Lewis, M. Raff, K. Roberts, and J.D. Watson, Molecular Biology of the Cell, 3rd ed. Garland, New York (1994), Chap. 10.

[5] S.J. Singer and G.L. Nicolson, Science, 175, 720 (1972).

[6] S. Ramaswamy and M. Rao, C. R. Acad. Sci. Paris IV, 2, 917 (2001).

[7] M. J. Saxton, K. Jacobson, Annu. Rev. Biophys. Biomol. Struct., 26, 373 (1997); M. J. Saxton, Int. J. Biochem., 22, 801 (1990).

[8] S. Levin and R. Korenstein, Biophys. J., 60, 733 (1991).

[9] S. Tuvia, A. Almagor, A. Bitler, S. Levin, R. Korenstein, and S. Yedgar, Proc. Natl. Acad. Sci. U.S.A., 94, 5045 (1997).

[10] M. Edidin, Annu. Rev. Biophys. Bioeng., 3, 179 (1974).

[11] J.-B. Manneville, P. Bassereau, S. Ramaswamy, and J. Prost, Phys. Rev. E, 64, 021908 (2001).

[12] R. Shlomovitz and N. S. Gov, Phys. Rev. Lett., 98, 168103 (2007).

[13] J. Zimmermann et al, Biophys. J, 102, 287 (2012).

[14] N. S. Gov, Phys. Rev. E, 73, 041918 (2006).

[15] A. Naji and F. L. H. Brown, J. Chem. Phys., 126, 235103 (2007).

[16] J. Pécréaux, H.-G. Döbereiner, J. Prost, J.-F. Joanny, and P. Bassereau, Eur. Phys. J. E, 13, 277 (2004).

[17] F.-C. Tsai, B. Stuhrmann and G. H. Koendrink, Langmuir, 27, 10061 (2011).

[18] R.A. Simha, S. Ramaswamy, Phys. Rev. Lett., 89, 058101 (2002).

[19] K. Kruse, J.F. Joanny, F. Julicher, J. Prost and K. Sekimoto, Eur. Phys. J. E, 16, 5 (2005).

[20] S. Ramaswamy, Annu. Rev. Cond. Matt. Phys., 1, 323 (2010).

[21] G.I. Menon, arXiv:1003.2032.

[22] J.-F. Joanny, J. Prost, in Biological Physics, Poincaré Seminar 2009, edited by B. Duplantier, V. Rivasseau (Springer, 2009) pp. 1-32.

[23] M. C. Marchetti et al, arXiv:1207.2929.

[24] G. Salbreux, J. Prost and J.-F. Joanny, Phys. Rev. Lett., 103, 058102 (2009).

[25] M. Mayer et al, Nature (London), 467, 617 (2010).

[26] J. S Bois, F. Jülicher and S. W. Grill, Phys. Rev. Lett., 106, 028103 (2011).

[27] A. Zumdieck, K. Kruse, H. Bringmann, A.A. Hyman and F. Jlicher PLoS ONE, 2, e696 (2007).

[28] H.-G. Döbereiner et al, Phys. Rev. Lett., 97, 38102 (2006).

[29] S. Sankararaman and S. Ramaswamy, Phys. Rev. Lett., 102, 118107 (2009).

[30] M. H. Köpf, S. V. Gurevich, T. Wulf and R. Friedrich, Phys. Rev. E, 83, 040601 (R) (2011).

[31] P. G. Saffman and M. Delbrück, Proc. Nat. Acad. Sci., USA, 72, 3111 (1975).

[32] P.-G. de Gennes and J. Prost, The Physics of Liquid Crystals, Clarendon, Oxford (1993).

[33] H.A. Stone, in Nonlinear PDEs in Condensed Matter and Reactive Flows, NATO Science Series C: Mathematical and Physical Sciences, 569, H. Berestycki and Y. Pomeau eds., Kluwer Academic, Dordrecht, The Netherlands (2002); A. Oron, S.H. Davis and S.G. Bankoff, Rev. Mod. Phys., 69, 931 (1997).

[34] T. C. Lubensky and F. C. MacKintosh, Phys. Rev. Lett., 71, 1565 (1993).

[35] Statistical Mechanics of Membranes and Surfaces, edited by D. Nelson, T. Piran, and S. Weinberg World Scientific, Singapore (1989).

[36] Hence for wavevector range given by $\eta q^{2} \sim \eta^{\prime} q / h_{0}$, i.e., when the ambient fluid is expected to become important, our results are not expected to hold.

[37] R. A. Simha and S. Ramaswamy, Phys. Rev. Lett., 89, 058101 (2002).

[38] Thermal noise $\xi_{\theta}$ has variance proportional to the inverse of the rotational viscosity, which we assume to be numerically same as $\eta$ for simplicity.

[39] See R. A. Simha and S. Ramaswamy, Phys. Rev. Lett., 89, 058101 (2002) for another (but not directly related) example where one obtains instability at long wavelengths for both signatures of a parameter analogous to $\Delta \mu$ in a system of coupled dynamics of fluctuations of concentration and local polar order in an ordered suspension of active polar particles.

[40] R. Reigada and J. Gomez, Biophysical Reviews and Letters, 5, 1 (2010).

[41] M. Bornens, M. Paintrand and C. Celati, J. Cell Biol., 109, 1071 (1989); O. J. Pletjushkina, Z. Rajfur, P. Pomorski, T. N. Oliver, J. M. Vasiliev and K. A. Jacobson, Cell Motil. Cytoskel., 48, 235 (2001); F. Jülicher and J. Prost, Phys. Rev. Lett., 78, 4510, (1997).

[42] S. Chiantia, J. Ries, and P. Schwille, BBA-Biomembranes, 1788, 225 (2009).

[43] P. H. M. Lommerse, H. P. Spaink, and T. Schmidt, BBA-Biomembranes, 1664, 119 (2004).

[44] E. A. J. Reits and J. J. Neefjes, Nat. Cell Biol., 3, E145 (2001).

[45] C. W. Cairo and D. E. Golan, Biopolymers, 89, 409 (2008). 
[46] H. Lamb, Hydrodynamics (Cambridge University Press, 1959).

[47] E. Reister-Gottfried, S. M. Leitenberger, and U. Seifert, Phys. Rev. E, 81, 031903 (2010).

[48] R. Voituriez, J.-F. Joanny and J. Prost, Euro. Phys. Lett., 70, 404 (2005).

[49] N. Sarkar and A. Basu, Eur. Phys. J. E, (2011).

[50] J. Toner and Y. Tu, Phys. Rev. E, 58, 4828 (1998).

[51] N. Sarkar and A. Basu, work in progress. 